\title{
Implementation and characterization of an RF ion funnel ion guide as a proton transfer reaction chamber
}

\author{
Phil A. Brown ${ }^{\mathrm{a}, \mathrm{b}, *}$, Simona M. Cristescu ${ }^{\mathrm{a}}$, Stephen J. Mullock ${ }^{\mathrm{c}}$, D. Fraser Reich ${ }^{\mathrm{c}}$, \\ Caroline S. Lamont-Smith ${ }^{\mathrm{c}}$, Frans J.M. Harren ${ }^{\mathrm{a}}$ \\ a Radboud University Nijmegen, Institute for Molecules and Materials (IMM), P.O. Box 9010, 6500 GL Nijmegen, The Netherlands \\ b TI-COAST, P.O. Box 18, 6160 MD Geleen, The Netherlands \\ ${ }^{\mathrm{c}}$ Kore Technology Ltd., Cambridgeshire Business Park, Ely, Cambridgeshire, CB7 4EA, UK
}

\section{A R T I C L E I N F O}

\section{Article history:}

Received 2 October 2016

Received in revised form

17 December 2016

Accepted 3 January 2017

Available online 5 January 2017

\begin{abstract}
A B S T R A C T
A radio frequency ion funnel reaction chamber is applied for use with a proton transfer reaction ion trap mass spectrometer (PIT-MS). The improvement in sensitivity over our dc-only drift tube ranged from $7.2 \mathrm{x}$ for isoprene to $65 \mathrm{x}$ for benzene. The ideal settings of the ion funnel are found to be a dc voltage of $60 \mathrm{~V}$, RF voltage of $240 \mathrm{~V}$ and a pressure of $1.80 \mathrm{mbar}$. An experimental and theoretical investigation of the ion funnel is presented. Simulations using SIMION and experimental measurements of the $m / z$ dependent transmission of the ion funnel are performed, as well as simulations of the trajectories of ions and their survival probability. These are used to deduce a greater understanding of the operation of the ion funnel and the sensitivity improvements. The funnel is shown to favor transmission of higher $m / z$, and to increase sensitivity by a combined effect of focusing and increased effective reaction time. The product ions of an example set of compounds are measured systematically and the reagent ion branching profiles studied and compared to ion trap fragmentations. This represents an important undertaking in the characterization studies of ion funnel technology for use in PTR-MS.
\end{abstract}

(C) 2017 Elsevier B.V. All rights reserved.

\section{Introduction}

Proton transfer reaction mass spectrometry (PTR-MS) is a technique capable of measuring trace concentrations of volatile organic compounds (VOCs) in real time [1,2]. The technique has applications in various scientific disciplines including atmospheric chemistry [3-5], food and aroma science [6-8], medical and biological applications [9-11] and security [12,13]. From the introduction of the technique in the 1990s [14] to the present day [15], improvements have been made to the technique: better compound identification using alternative types of mass spectrometry, Time of Flight Mass spectrometry [16,17] and Ion Trap Mass Spectrometry [18], novel sampling methods [19-21], and sensitivity improvements $[15,22]$. Sensitivity improvements offer obvious advantages in all of the above mentioned areas; whether it provides potential new biomarkers in breath analysis or reduces the limit of detection for threat agents, the potential improvements are clear and applicable.

\footnotetext{
* Corresponding author at: Radboud University Nijmegen, Institute for Molecules and Materials (IMM), P.O. Box 9010, 6500 GL Nijmegen, The Netherlands.

E-mail address: p.brown@science.ru.nl (P.A. Brown).
}

Increasing sensitivity in PTR-MS by using ion funnel technology has in recent years been a pursued topic of ingenuity [22-25]. Herein the characterization and sensitivity improvements gained using an RF ion funnel as proton transfer reaction chamber are presented. Here we present a funnel that has been developed at Radboud University, based on a design by Kore Technology Ltd (Ely, UK), published in collaboration with[22]. The PTR-MS used for testing the funnel is an in-house constructed Proton Transfer Reaction Ion Trap Mass Spectrometer (PIT-MS) [18]. Before, the reaction chamber of the PIT-MS was a standard direct current (dc) drift tube, a commonly used component of PTR-MS technology since its inception [14].

In the PTR-MS reaction chamber $\mathrm{H}_{3} \mathrm{O}^{+}$ions transfer a proton to a neutral VOC for later detection by the mass spectrometer unit. The conventional drift tube consists of a series of stacked electrodes $\sim 10 \mathrm{~cm}$ long, physically isolated from each other but electrically connected through a series of common resistors. This creates a consistent electric field of $40-60 \mathrm{~V} \mathrm{~cm}^{-1}$ when a voltage of $400-600 \mathrm{~V}$ is applied across the drift tube. Conventionally, the operating parameters of a drift tube are summarized as the ratio of the electric field $(E)$ and number density $(N)$; the reduced electric field $(E / N)$. For a PTR-MS drift tube, this value is varied between 80 and $160 \mathrm{Td}$ (1 $\mathrm{Td}=10^{-17} \mathrm{~V} \mathrm{~cm}^{2}$ ). 


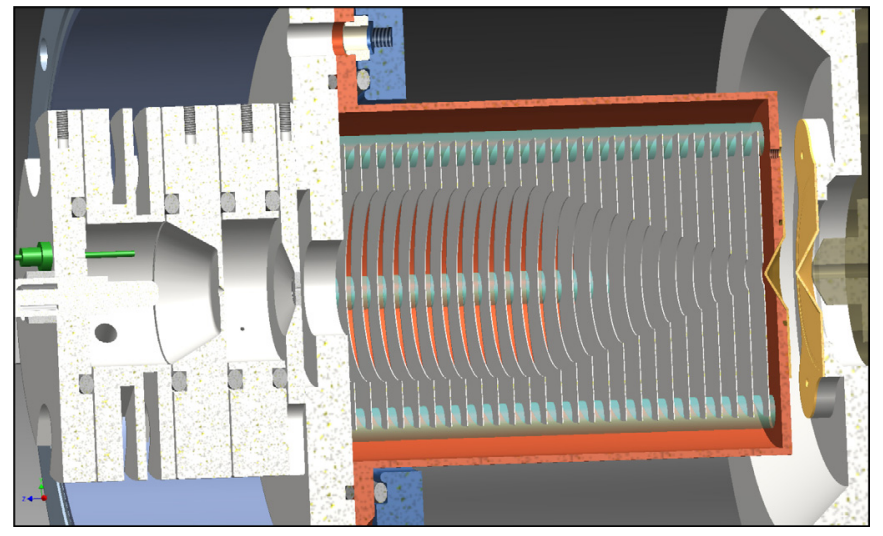

Fig. 1. 3-D design image of ion funnel implementation. The ion funnel is series of 27 plates, mounted on PEEK rods and housed in a vacuum chamber (red). Ion source and skimmers are also shown. (For interpretation of the references to colour in this figure legend, the reader is referred to the web version of this article.)

Ion funnels themselves have been implemented as common components in mass spectrometry since their initial introduction in the 1990s [26-28]. The basic tenet of an ion funnel is to focus ions in intermediate pumping regions at pressures too high to effectively use multipole ion guides. The pressures mentioned are in the mbar range; the same pressure range used in the drift tube of a PTR-MS and, therefore, an obvious potential exists to exploit the technology for use as a PTR-MS reaction chamber. This publication comments on the successful exploitation of ion funnel approach with PTR-MS, showing sensitivity improvements, effective transmission of the ions, protonated water cluster abundance and product ion branching ratios. The work provides confirmatory evidence of the work of Barber et al. [22] and further details of the mechanism of enhancement via simulations. A software package (SIMION 8.0.4) for simulation of ion trajectories in the presence of electric fields is used to calculate the potential behavior of the ion funnel. A comparison is made between the SIMION model and the experimental parameters of the actual funnel.

\section{Experimental methods}

\subsection{Experimental funnel}

The mechanical design of the funnel (see Fig. 1) consists of a $92 \mathrm{~mm}$ long, grounded housing in which 27 stainless steel plates (outer diameter $60 \mathrm{~mm}$, width $0.2 \mathrm{~mm}$ ) are set equally spaced $(3.2 \mathrm{~mm})$. The first 13 plates have an inner hole diameter of $40 \mathrm{~mm}$; the latter half of the funnel plates tapers the inner diameter of the plates down to $4.6 \mathrm{~mm}$ at the final plate; a $1.6 \mathrm{~mm}$ change in internal diameter for adjacent plates. Adjacent plates over the whole funnel are connected by $1 \mathrm{M} \Omega$ resistors and each adjacent tapering plate is connected to a capacitor chain of $220 \mu \mathrm{F}$ capacitors to provide a balanced RF voltage. Vacuum feedthroughs provide electrical connections to the first and last plate. Two further vacuum feedthroughs provide connections to the even numbered plates between 14 and 22, and odd numbered plates between 15 and 23, with two additional feedthroughs for even Plates 24 and 26 and odd Plates 25 and 27. The final four plates of the funnel are operated with a reduced RF voltage; the reducing factor being approximately 0.6 . Typical dc voltages range from 40 to $120 \mathrm{~V}$ and the typical RF voltage range is $180-330 \mathrm{~V}_{\mathrm{pp}}$ at $820 \mathrm{kHz}$. The pressure investigated inside the funnel is between $1.40-2.20$ mbar.

The funnel is characterized mostly using a 1 ppmv calibration mixture in nitrogen of methanol, acetaldehyde, acetone, isoprene, benzene, toluene, o-xylene and $\alpha$-pinene (Linde Gas, Dieren, the Netherlands). The compounds were chosen to represent a range of

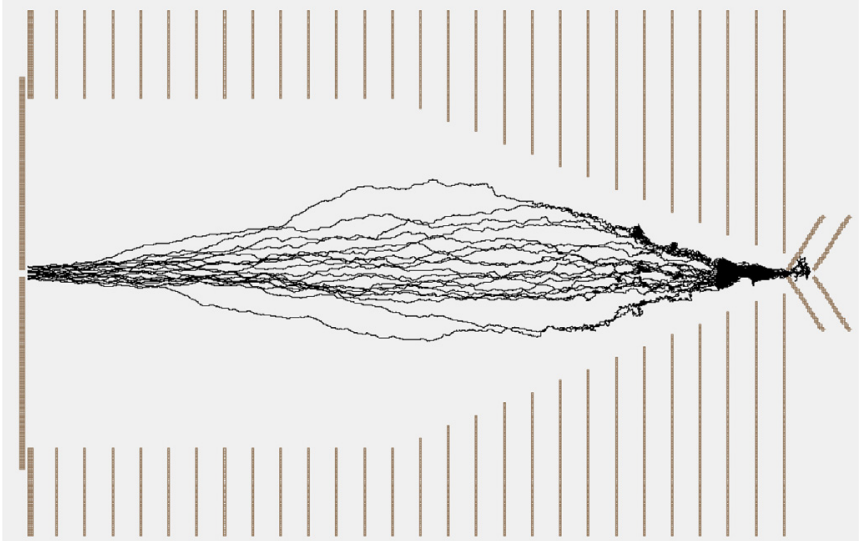

Fig. 2. Ion trajectory calculated using SIMION 8.0 .4 for $m / z 107$ with $R F=240 \mathrm{~V}$, $\mathrm{f}=820 \mathrm{kHz}, \mathrm{dc}=60 \mathrm{~V}$ and buffer gas pressure $1.80 \mathrm{mbar}$.

masses and chemical classes. This mixture can be further diluted as necessary by mixing with clean nitrogen using calibrated mass flow controllers (Brooks Instrument, Ede, the Netherlands). The characteristics of the ion funnel on sensitivity, transmission of the ions, protonated water cluster abundance and product ion branching ratios are investigated. By changing the ion funnel pressure and the $\mathrm{RF}$ and dc voltages of the funnel the sensitivity is investigated. Furthermore, specific gas mixtures to measure the transmission of the funnel are made by flowing clean nitrogen above a sample liquid in question, to extract a headspace flow for introduction to the PIT-MS. For this liquid samples of acetonitrile, butane-2,3-dione, methyl benzoate, and methyl 2-hydroxybenzoate were used (purity $>99 \%$, Sigma-Aldrich, New Jersey, USA). Studies of fragmentation are performed on isolated compounds by creating a vapor headspace in a flow of nitrogen above the liquid compound and further diluting this with clean nitrogen. The compounds used for this fragmentation study were acetic acid, toluene, xylene and alpha-pinene (>99\% purity, Sigma Aldrich, New Jersey, USA). The concentration was set such that dimerization was not a concern.

\subsection{Mathematical modeling of the ion funnel}

To study the ion funnel a mathematical model was constructed using SIMION. The ion trajectory modeling software simulates collisions using the hard-sphere, elastic, ion-neutral collision model available with SIMION 8.0.4 (Scientific Instrument Services, Inc., NJ, USA) [29]. A geometry (GEM) file is built to define the electrode geometry and a subsequent file is written in the Lua programming language to control the RF and dc voltages of the funnel, along with setting ion and buffer gas parameters (shown in Supplementary Material, Program S 1). As an example, an ion trace is shown in Fig. 2 using SIMION for our ion funnel design with an RF voltage $240 \mathrm{~V}, \mathrm{f}=820 \mathrm{kHz}$, dc voltage $=60 \mathrm{~V}$ and pressure $=1.80 \mathrm{mbar}$ for an ion of $m / z 107$, corresponding to o-xylene. The model allowed for Coulomb repulsion between ions to be incorporated. All other parameters of the simulation are explained in the Lua file in the supplementary material. The ion trajectories diverge as the ions are randomly scattered away from their normal trajectories by collisions with the buffer gas.

\subsection{Measurements}

The transmission of the funnel is measured by introducing a high concentration of single analyte to the PIT-MS and measuring the decrease in $\mathrm{H}_{3} \mathrm{O}^{+}$and corresponding increase in the protonated monomer product ion. By this means a mass dependent transmission curve relative to $\mathrm{m} / \mathrm{z} 19$ can be calculated. This procedure is 
followed, both for the new ion funnel and for the old, conventional drift tube, using the PIT-MS. For the ion funnel the compounds used were; acetonitrile $(\mathrm{m} / \mathrm{z} 42)$, butane-2,3-dione $(\mathrm{m} / \mathrm{z} 87)$, methyl benzoate $(m / z 137)$ and methyl salicylate $(m / z 153)$. For the old, conventional drift tube, methyl salicylate was not used, but acetone $(m / z 59)$ was. In the conventional drift tube, methyl salicylate was found to result in a fragment ion with greater than $5 \%$ product ion branching ratio. Within the mathematical approach using SIMION, the mass dependent transmission curve of the funnel is calculated simply comparing the modeled transmission of ions at $m / z 42,87,137$ and 153 and normalizing them on $\mathrm{H}_{3} \mathrm{O}^{+}$ions at $m / z$ 19 . These models are made, using a dc voltage of $60 \mathrm{~V}, \mathrm{RF}$ voltage of $240 \mathrm{~V}$ and a pressure of $1.80 \mathrm{mbar}$.

The experimental characterization of the sensitivity of the ion funnel is performed using the 1 ppmv calibration mixture (calmix) of 8 VOCs in nitrogen (methanol, acetaldehyde, acetone, isoprene, benzene, toluene, 0 -xylene and $\alpha$-pinene). The signal intensity is recorded for each protonated monomer while the various settings of the ion funnel are altered; pressure (1.40-2.20 mbar), RF voltage (180-330 V), dc voltage (20-80 V). This testing range was decided upon after preliminary measurements found that these settings approximate the best operating conditions.

For comparison, characterization has been performed of a standard drift tube using a quadrupole PTR-MS; this system has been previously developed at Radboud University [30] and is closely comparable in operating principle to the instrument described by Lindinger et al. [2] on which commercial devices are based [31]. This drift tube (93 mm long) is operated at a pressure of $1.85 \mathrm{mbar}$ and at a range of electric fields of $43-65 \mathrm{~V} \mathrm{~cm}^{-1}$; this produces a reduced electric field range of $106-160 \mathrm{Td}$. For the ion funnel a constant pressure of 1.80 mbar is selected and the RF and dc voltage changed between 180 and $330 \mathrm{~V}$ and $20-80 \mathrm{~V}$ respectively.

To study the behavior of the funnel, molecular fragmentation is investigated in the ion funnel (in combination with the ion trap) and compared with the standard drift tube using the quadrupole PTR-MS. Fragmentation behavior is corrected for the measured transmission curve, both for the standard drift tube using the quadrupole PTR-MS and for the PIT-MS; the data are presented as product ion branching ratios, as outlined by Brown et al. [32].

To calculate sensitivity, the calibration mixture was diluted in five steps. The signal intensity of the protonated monomer only is normalized to the sum of signals for the $\mathrm{H}_{3} \mathrm{O}^{+}$ion and the protonated water dimer, and to a normalization factor $1 \times 10^{6}$ [33]. These normalized values are then plotted against their corresponding concentration to calculate a calibration curve. The slope of this curve is found with a least squares fitting procedure and provides the response of the system against an applied concentration, which is the sensitivity of the system. The limit of detection is taken as 3 times the standard deviation of the signal (in concentration) when clean nitrogen is introduced in the system. Although molecular ion fragmentation is considered and investigated, fragment ions are not included in calculations of sensitivity.

\section{Results}

The achieved sensitivity of the ion funnel in combination with the PIT-MS is shown in Table 1, and compared to the sensitivity achieved with the standard drift tube and PIT-MS, operated with conditions at $119 \mathrm{Td}$. The ion funnel is operated with a dc voltage of $60 \mathrm{~V}$, RF voltage of $240 \mathrm{~V}$ and a pressure of $1.80 \mathrm{mbar}$. As can be seen in Table 1, a significant sensitivity improvement is observed using the funnel, most notably for benzene $(65 x)$, toluene $(43 x)$ and o-xylene $(41 \mathrm{x})$. The observed strong increase in sensitivity for benzene can be explained from the fact that the ion funnel results in a higher $\mathrm{H}_{3} \mathrm{O}^{+}$intensity owing to break up if the protonated water clusters compared to the dc mode at the same drift tube voltage. Benzene is non-reactive with protonated water clusters [33].

The transmission of the ion funnel was calculated using SIMION and found to be linear with increasing $\mathrm{m} / \mathrm{z}$ of the ion (Fig. 3b). The measured transmission of the funnel with PIT-MS was found to increase in a non-linear manner, approaching a quadratic increase (Fig. 3a). Using a conventional drift tube with the PIT-MS the transmission is measured to be a linearly increasing transmission with increasing mass (Fig. 3c). Shamlouei et al. show a decreasing transmission with increasing mass of the ion due to increasing drift time for ions of higher $m / z$ [34]. That the measured transmission of the drift tube appears atypical indicates that the transmission of the ion trap is favorable to higher $m / z$ ions. This agrees with a theoretical understanding of ion traps, where heavier ions can be more efficiently trapped, and thus measured. The measured transmission of the ion funnel is thought to be due to a superposition of the ion funnel and ion trap transmissions, as shown in Fig. 3a.

In Fig. 4 the effect of changing the dc electric field in the ion funnel is illustrated; the dc voltage was varied between 15 and $180 \mathrm{~V}$; RF voltage $=200 \mathrm{~V}$, pressure $=1.60 \mathrm{mbar}$. In Fig. $4 \mathrm{a}$ intensity values are recorded for the primary water ion $(m / z 19)$ next to specific masses within the calibration mixture (acetaldehyde $m / z 45$, acetone $m / z 59$, isoprene $m / z 69$, benzene $m / z 79$, toluene $m / z 93$, o-xylene $m / z 107$, and $\alpha$-pinene $m / z 137$ ) at a concentration of 465 ppbv. A decrease in the observed product ion signal is viewed as the applied dc field is increased from $60 \mathrm{~V}$ onwards. For all product ions the change in intensity over the range $60-180 \mathrm{~V}$ is an order of magnitude decrease. For the $\mathrm{H}_{3} \mathrm{O}^{+}$ion a shallower decrease is observed over this range. At the lower voltages of the tested dc field, all ions show decreased signal of similar magnitude. Fig. 4c shows the calculated ion transmission for $m / z 107$ from the model, at pressure 1.60 mbar and RF voltage $200 \mathrm{~V}$. Fig. $4 \mathrm{~b}$ shows from the model a strong increase in the lifetime for the $m / z 19$ ion at lower dc voltages.

The experimental sensitivity of the ion funnel is displayed in Fig. 5. The $\mathrm{H}_{3} \mathrm{O}^{+}$ions are shown along with acetone and benzene signals. The other 5 compounds in the calibration mixture are shown similarly in the Supplementary Material, Fig. S 1. For a constant concentration of the input analyte, the intensity of the primary product ions is displayed as a function of dc and RF voltages, as well as the pressure, producing a 3-D plot. The color of each point represents an intensity as shown in the color bar, right of each plot

Table 2 shows the fragmentation data for the compounds alphapinene, acetic acid, o-xylene and toluene in the PTR-MS with standard drift tube and PIT-MS ion funnel. The $m / z$ and likely chemical formula for each product ion is presented, along with the percentage product ion branching ratio. Funnel measurements are all made at $1.80 \mathrm{mbar}$, with dc voltage of $80 \mathrm{~V}$ and drift tube measurements at 1.85 mbar. Branching ratios for the reagent ion signal $\left(\mathrm{H}_{3} \mathrm{O}^{+}\right.$and $\left.\mathrm{H}_{3} \mathrm{O}^{+} \cdot \mathrm{H}_{2} \mathrm{O}\right)$ are also presented.

\section{Discussion}

The ion funnel exhibited here shows significant sensitivity improvement over the previously installed conventional drift tube (Table 1). This confirms the earlier work of Barber et al. who report a 1-2 order of magnitude increase in sensitivity across a range of compounds studied (acetaldehyde 45x, acetone 200x) [22]. This work has improved the understanding of the observed increase in sensitivity through trajectory modeling using SIMION. Their group report a 1-2 order of magnitude increase in sensitivity across a range of compounds studied (acetaldehyde $45 x$, acetone $200 x$ ). In the implementation of our ion funnel the reduced electric field $(\mathrm{E} / \mathrm{N})$ in the initial half of the reaction chamber (dc only section) is less than $30 \mathrm{Td}$, while in the case of Barber et al. the initial half 
Table 1

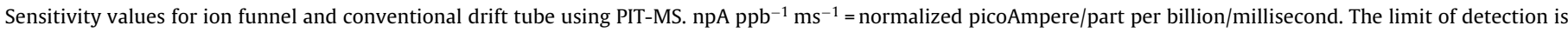
taken 3 times standard deviation of the signal (in concentration) when clean nitrogen is introduced in the system.

\begin{tabular}{|c|c|c|c|c|}
\hline Compound $(m / z)$ & Ion Funnel npA ppb ${ }^{-1} \mathrm{~ms}^{-1}$ & Drift Tube npA ppb ${ }^{-1} \mathrm{~ms}^{-1}$ & Sensitivity ratio Ion funnel/Drift tube & Limit of detection Ion funnel (ppb) \\
\hline Acetaldehyde (45) & 3040 & 330 & 9.2 & 8.8 \\
\hline Acetone (59) & 46000 & 1150 & 40 & 4.0 \\
\hline Isoprene (69) & 2520 & 340 & 7.3 & 2.9 \\
\hline Benzene (79) & 11840 & 180 & 65 & 0.38 \\
\hline Toluene (93) & 42460 & 1000 & 43 & 0.30 \\
\hline o-Xylene (107) & 89100 & 2180 & 41 & 0.15 \\
\hline Alpha-pinene (137) & 39940 & 1700 & 23 & 0.26 \\
\hline
\end{tabular}

a)

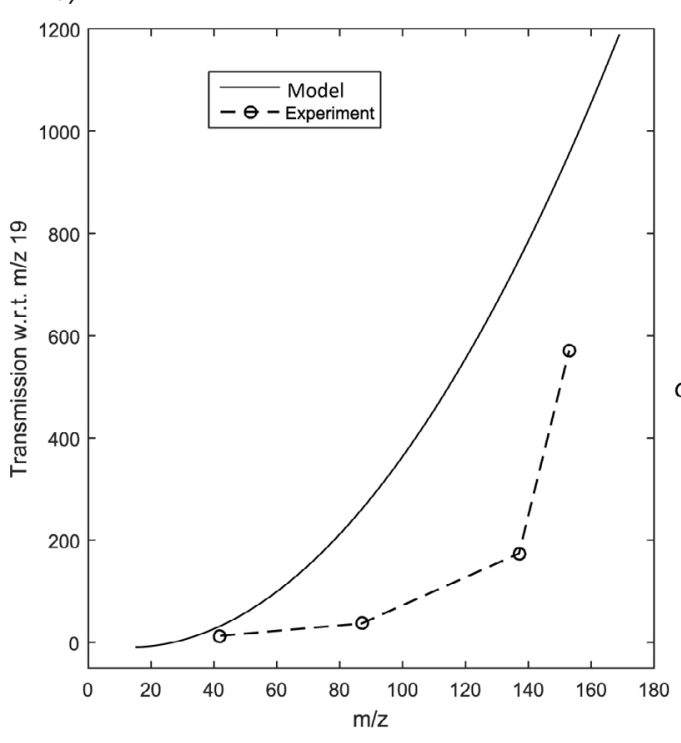

b)
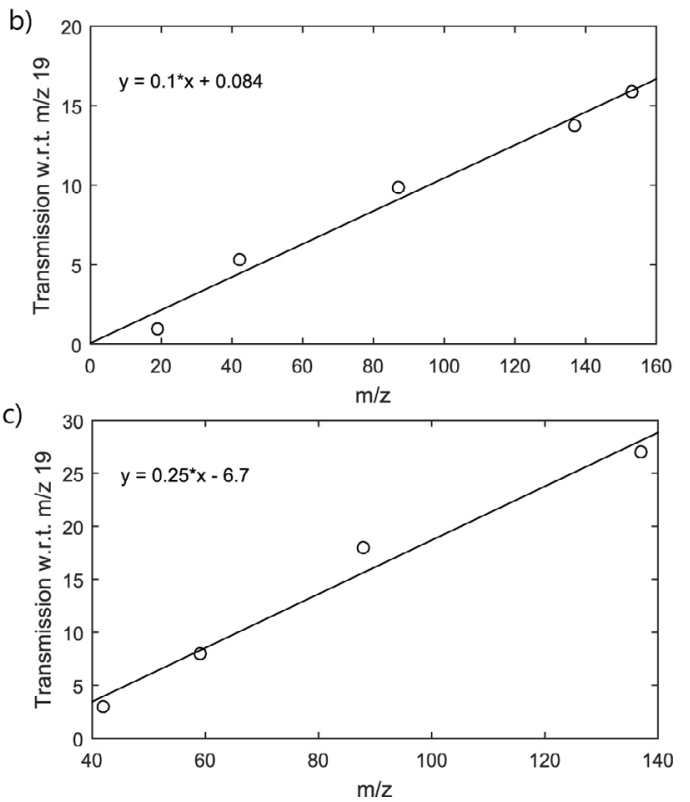

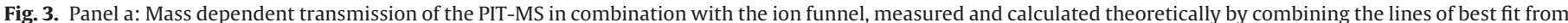

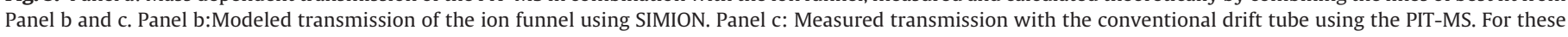
measurements all transmission is shown with respect to (w.r.t.) $m / z 19$.
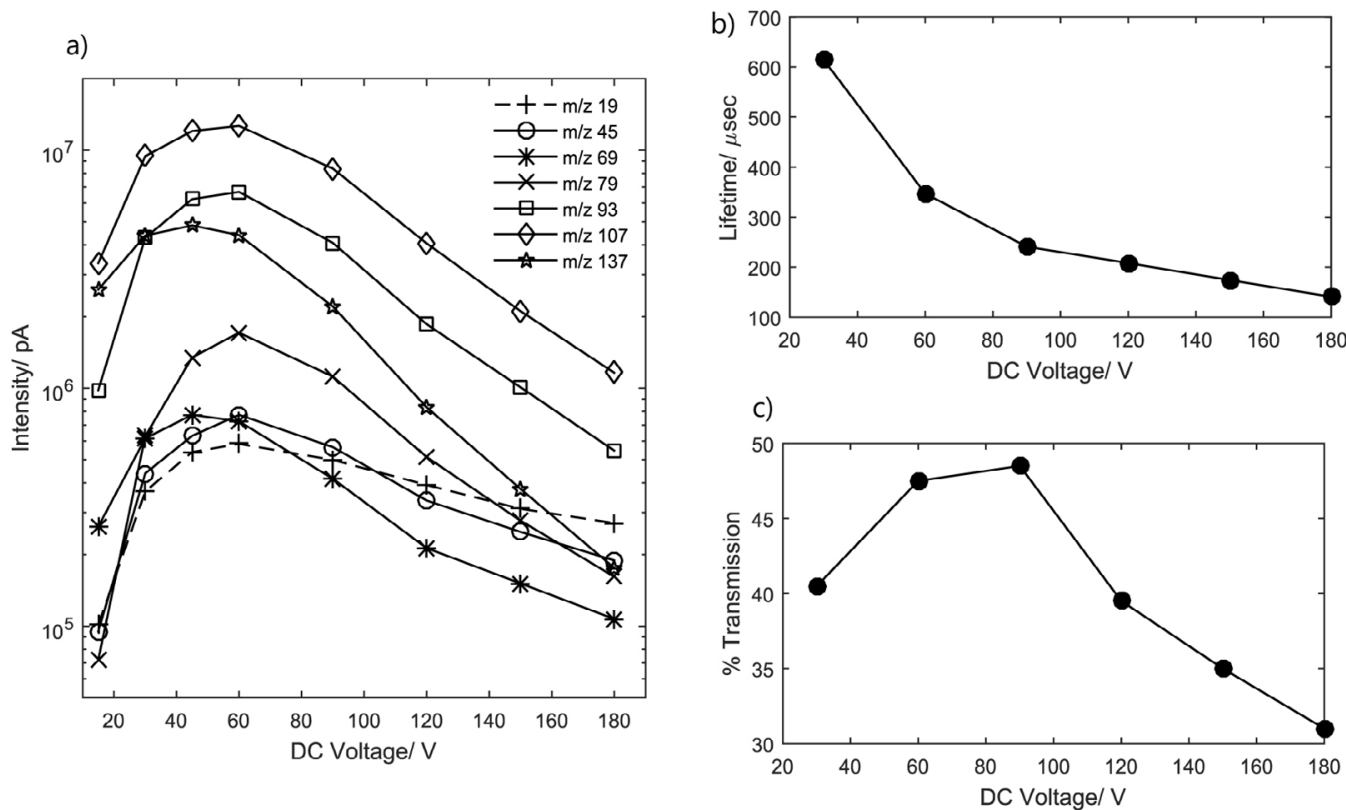

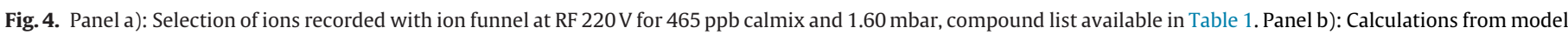

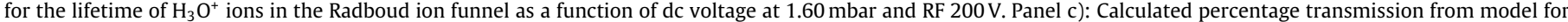
$\mathrm{m} / \mathrm{z} 107$ as a function of dc voltage at 1.60 mbar and RF $200 \mathrm{~V}$. 
Table 2

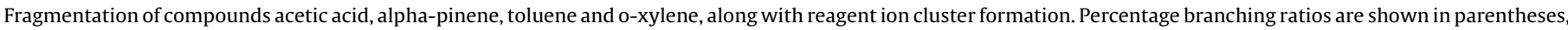
$m / z$ shown in plain text and product ion chemical formula given alongside.

\begin{tabular}{|c|c|c|c|c|}
\hline & \multicolumn{4}{|c|}{ Product Ions and Branching Ratios (\%) } \\
\hline & Drift tube & & Ion funnel dc $80 \mathrm{~V}$ & \\
\hline \multirow{3}{*}{$\begin{array}{l}\text { Chemical name } \\
\text { Reagent ion }\end{array}$} & $106 \mathrm{Td}$ & $160 \mathrm{Td}$ & RF $180 \mathrm{~V}$ & RF $330 \mathrm{~V}$ \\
\hline & $19(36) \mathrm{H}_{3} \mathrm{O}^{+}$ & $19(97) \mathrm{H}_{3} \mathrm{O}^{+}$ & $19(80) \mathrm{H}_{3} \mathrm{O}^{+}$ & $19(95) \mathrm{H}_{3} \mathrm{O}^{+}$ \\
\hline & $37(64) \mathrm{H}_{3} \mathrm{O}^{+} \cdot \mathrm{H}_{2} \mathrm{O}$ & $37(3) \mathrm{H}_{3} \mathrm{O}^{+} \cdot \mathrm{H}_{2} \mathrm{O}$ & $37(20) \mathrm{H}_{3} \mathrm{O}^{+} \cdot \mathrm{H}_{2} \mathrm{O}$ & $37(5) \mathrm{H}_{3} \mathrm{O}^{+} \cdot \mathrm{H}_{2} \mathrm{O}$ \\
\hline \multirow[t]{3}{*}{ Alpha-pinene } & $137(54) \mathrm{MH}^{+}$ & $137(25) \mathrm{MH}^{+}$ & $137(67) \mathrm{MH}^{+}$ & $137(61) \mathrm{MH}^{+}$ \\
\hline & 95 (1) $\mathrm{MH}^{+}-\mathrm{C}_{3} \mathrm{H}_{6}$ & 95 (7) $\mathrm{MH}^{+}-\mathrm{C}_{3} \mathrm{H}_{6}$ & $95(7) \mathrm{MH}^{+}-\mathrm{C}_{3} \mathrm{H}_{6}$ & 95 (14) $\mathrm{MH}^{+}-\mathrm{C}_{3} \mathrm{H}_{6}$ \\
\hline & $81(45) \mathrm{MH}^{+}-\mathrm{C}_{4} \mathrm{H}_{8}$ & $81(68) \mathrm{MH}^{+}-\mathrm{C}_{4} \mathrm{H}_{8}$ & $81(26) \mathrm{MH}^{+}-\mathrm{C}_{4} \mathrm{H}_{8}$ & $81(25) \mathrm{MH}^{+}-\mathrm{C}_{4} \mathrm{H}_{8}$ \\
\hline \multirow[t]{2}{*}{ Acetic Acid } & $61(82) \mathrm{MH}^{+}$ & $61(37) \mathrm{MH}^{+}$ & $61(93) \mathrm{MH}^{+}$ & $61(81) \mathrm{MH}^{+}$ \\
\hline & $43(17) \mathrm{MH}^{+}-\mathrm{H}_{2} \mathrm{O}$ & $43(62) \mathrm{MH}^{+}-\mathrm{H}_{2} \mathrm{O}$ & $43(7) \mathrm{MH}^{+}-\mathrm{H}_{2} \mathrm{O}$ & 43 (19) $\mathrm{MH}^{+}-\mathrm{H}_{2} \mathrm{O}$ \\
\hline \multirow[t]{5}{*}{ Toluene } & $93(100) \mathrm{MH}^{+}$ & $93(91) \mathrm{MH}^{+}$ & $93(76) \mathrm{MH}^{+}$ & $93(69) \mathrm{MH}^{+}$ \\
\hline & & $95(2)$ & $95(11)$ & $95(15)$ \\
\hline & & $\mathrm{MH}^{+}-\mathrm{CH}_{4}+\mathrm{H}_{2} \mathrm{O}$ & $\mathrm{MH}^{+}-\mathrm{CH}_{4}+\mathrm{H}_{2} \mathrm{O}$ & $\mathrm{MH}^{+}-\mathrm{CH}_{4}+\mathrm{H}_{2} \mathrm{O}$ \\
\hline & & $91(5) \mathrm{MH}^{+}-\mathrm{H}_{2}$ & 91 (12) $\mathrm{MH}^{+}-\mathrm{H}_{2}$ & $91(16) \mathrm{MH}^{+}-\mathrm{H}_{2}$ \\
\hline & & 77 (2) $\mathrm{MH}^{+}-\mathrm{CH}_{4}$ & & \\
\hline \multirow[t]{2}{*}{ o-xylene } & $107(100) \mathrm{MH}^{+}$ & $107(100) \mathrm{MH}^{+}$ & 107 (89) $\mathrm{MH}^{+}$ & $107(84) \mathrm{MH}^{+}$ \\
\hline & & & $91(11) \mathrm{MH}^{+}-\mathrm{CH}_{4}$ & $91(16) \mathrm{MH}^{+}-\mathrm{CH}_{4}$ \\
\hline
\end{tabular}

is at $\sim 60$ Td [22]. In general, typical reduced electric fields for PTRMS drift tubes are in between 90 and $140 \mathrm{Td}$ [32]; at the low end of this range water cluster formation becomes problematic and sensitivity to benzene in particular is greatly reduced $[33,35]$. In Table 1 a strong sensitivity enhancement for benzene is observed with the ion funnel, as compared with the other compounds measured. This shows that a reasonably high purity of $\mathrm{H}_{3} \mathrm{O}^{+}$, without water clusters, must be present in the reaction chamber. The dc only section of the funnel would have a high amount of water clusters, and subsequently, water cluster reactions. As such, the high $\mathrm{H}_{3} \mathrm{O}^{+}$monomer purity will be in the $\mathrm{RF}$ region, where a significant $\mathrm{H}_{3} \mathrm{O}^{+}$monomer-based proton transfer occurs.

Although not possessing the same reactivity characteristics as benzene, it is shown that the other VOCs also have a reduced reactivity in an environment with water clusters [35]. Bearing this information in mind, it is prudent to suggest that the dc-only section of the reaction chamber performs no obvious function in its current low reduced electric field implementation, with the primary proton transfer reaction and focusing both occurring in the funnel region. In a future design, ion funnel reaction chambers may be better designed without this feature of the dc-only section. Significant improvements in overall ion transmission may be possible without the potential ion divergence in the low reduced electric field region (see Fig. 2). Jordan et al. employ their ion funnel to only focus ions as they approach the exit of the drift tube. The dc only section of their funnel is at a reduced electric field of the same function as a standard drift tube [24].

It is noted that the improvement in acetone sensitivity is higher than either of the compounds at similar $m / z$ (isoprene and acetaldehyde). The reason for this improvement for acetone sensitivity may be that acetone is typically more reactive with the hydronium water cluster than the other compounds, and thus some proton transfer may occur in the low field dc only region of the ion funnel, and that the acetone may be boosted by the long reaction time such a low dc field would afford.

The greatest sensitivity is observed for each product ion (Fig. 5 and Fig. S 1) in the region between 40 and $60 \mathrm{~V} \mathrm{dc}$ and 180 and $300 \mathrm{~V}$ RF. At lower voltages it is believed that the focusing effect of the funnel is lost and ions transfer less efficiently. At higher voltages it may be tempting to suggest that fragmentation causes a reduction in sensitivity; this however is not seen in the results. For alpha pinene, at 1.80 mbar across a voltage range between 20 and $80 \mathrm{~V} \mathrm{dc}$ and $180-330 \mathrm{~V}$ RF, little change in branching ratio is observed, as shown in Table 2.
The aim of Fig. 3 is to explore the transmission of the ion funnel by simulation. The simulation (Fig. 3b) shows that the transmission increases linearly at higher $m / z$. To explore the validity of this simulation the transmission of the ion funnel was measured experimentally, but this measurement necessitates inclusion of the transmission of the ion trap. To take account of this, the transmission of the conventional drift tube is taken as a guide to the transmission of the ion trap. The superposition of the transmission of the ion funnel simulation and the measured ion trap produces the theoretical curve shown in Fig. 3a. The experimental curve can be seen to lag the transmission of the theoretical curve. This may be due to a number of factors. The measurement technique used does not take into account proton transfer reactions which may occur from the hydrated hydronium ion, and although the compounds chosen were done so to minimize fragmentation, some low percentage abundance fragment ions were observed ( $<5 \%$ of product ion branching ratio). The simulation itself assumes the ions originate at a single point, on the central axis, at the start of the ion funnel. However, the ions will themselves be produced at any point along the length of the ion funnel, and at varying distances from the central axis.

The shape of the measured transmission curve in Fig. 3a is nonlinear. This shape, as shown by the theoretical curve, can be explained as the superposition of two transmission curves, from the ion funnel and ion trap. The result of Fig. 3 also shows that the simulated transmission of the ion funnel would seem to be a reasonable guide to its actual transmission. However, a fuller investigation of the transmission of the ion funnel would be needed to confirm this.

Fig. 4a shows that, at dc voltages above $60 \mathrm{~V}$, there is a trend to lower signal intensity for all product ions, this trend appears independent of the mass of the product ions. Fig. $4 \mathrm{~b}$ and $4 \mathrm{c}$ show the contribution to this measured change in intensity from two different factors calculated from the model; ion focusing (as calculated for $m / z 107$ in Fig. 4c) and residence time for $\mathrm{H}_{3} \mathrm{O}^{+}$ions (Fig. 4b). A combination of these factors contribute to the change in signal intensity as a function of dc voltage; better focusing produces intuitively higher signal intensity, while a longer residence time of the (amongst others $\mathrm{H}_{3} \mathrm{O}^{+}$) ions allows more time for the proton transfer reaction to take place, improving product ion yield. In Fig. 4a, the $m / z 19$ ion $\left(\mathrm{H}_{3} \mathrm{O}^{+}\right)$shows the focusing effect, while the product ions express the combined effect of focusing and increased interaction time. The increase in interaction time is due to the oscillatory trajectory of the ions under influence of the RF electric field, this 

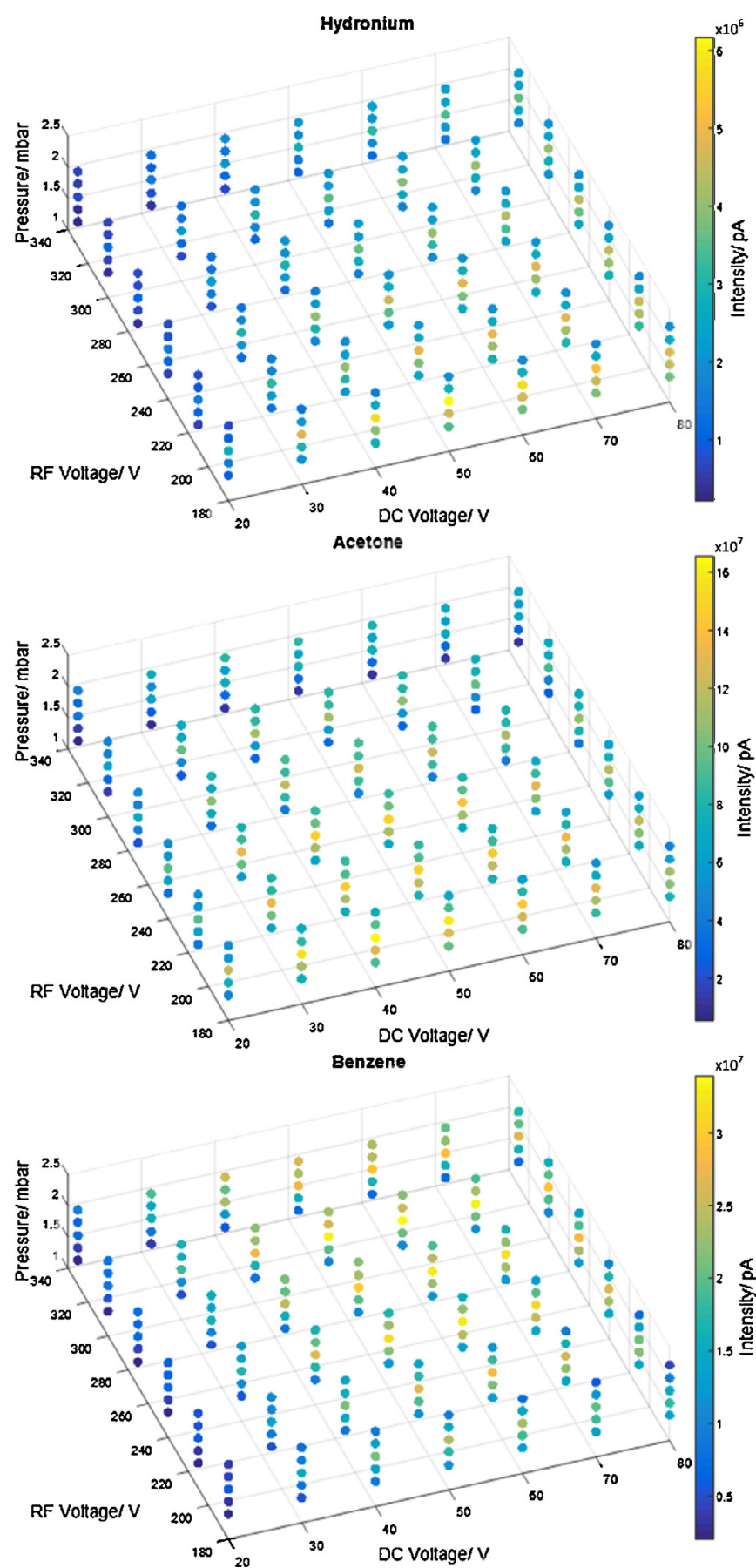

Fig. 5. Series of 3-D plots showing the intensity of ion signals, detected at constant concentration of the input analyte, for the combined effect of changing ion funnel pressure, dc voltage and RF voltage for $m / z 19$ hydronium, $m / z 59$ acetone and $m / z$ 79 benzene.

causes ions to undertake a longer path length. The loss in sensitivity at high dc voltages could be due to an effect on the transmittance of ions through the final end of the funnel, with ions perhaps becoming trapped and/or unstable in the funnel and colliding with the plates.

Similar to alpha-pinene, for acetic acid only a slight change is observed in fragmentation over the range of tested voltages for the ion funnel (see Table 2). With the conventional drift tube fragmentation is quite substantial. In accordance with our results, Christian et al. [36] found a $30 \%$ branching ratio for $m / z 43$ as a product ion of acetic acid at 2 mbar and 130 Td. Maleknia et al. [37] reported the

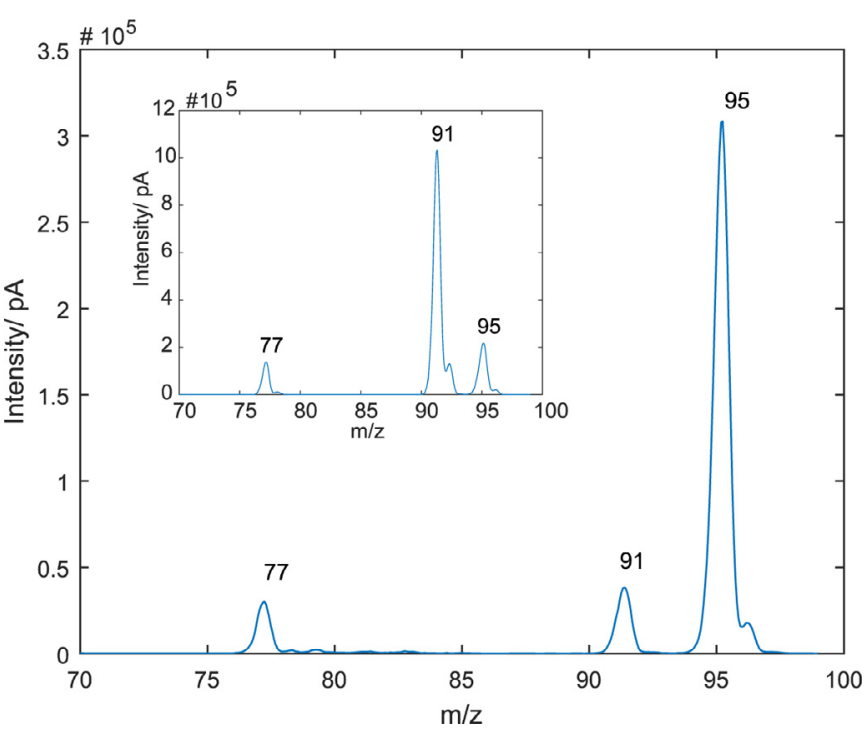

Fig. 6. Trapped ( $3 \mathrm{~ms}$ ) and fragmented at $8 \mathrm{~V}$ excitation voltage $(15 \mathrm{~ms}) \mathrm{m} / z$ 93 protonated monomer of toluene (inset), trapped $(3 \mathrm{~ms})$ and held in trap for $15 \mathrm{~ms}$ at $0 \mathrm{~V}$ excitation voltage ion at $\mathrm{m} / \mathrm{z} 77$ from inset spectrum (main figure).

$m / z 61$ product ion at an abundance of $4 \%$ in comparison to the $m / z$ 43 ion at a reduced electric field of between 124 and $160 \mathrm{Td}$.

For toluene in the standard drift tube, fragmentation was only observed at the highest reduced electric field setting of $160 \mathrm{Td}$. This agrees with literature where no fragmentation for toluene is reported with PTR-MS [35]. However, with the ion funnel significant branching ratios are recorded with fragment ions at 95 and $91 \mathrm{Da}$.

In the case of o-xylene, also no fragmentation is reported in the literature for standard drift tube measurements, similar to our observations (Table 2), but fragmentation to an $m / z 91$ fragment ion was observed in the ion funnel. This fragment ion is well known in mass spectrometry and indeed is observed as the most intense signal in the electron impact mass spectra of xylenes. Again these branching ratios remain reasonably consistent across the range of voltages tested, but in contrast to acetic acid and alpha-pinene a greater abundance of fragment ions is observed in the ion funnel than in the standard drift tube at $160 \mathrm{Td}$.

Worthy of investigation is the production of an $m / z 95$ fragment ion from the reaction of the hydronium ion with toluene (mass $92 \mathrm{Da}$ ) as this ion is heavier than the protonated monomer. Fig. 6 (insert) shows the recorded mass spectrum for the trapping and fragmentation of the protonated monomer of toluene, along with the mass spectrum from the trapping of the subsequent $m / z 77$ fragment ion (main figure). Both spectra are taken with the ion funnel. That trapping of $m / z 77$ results in a major ion at $m / z 95$ suggests that an association reaction occurs in the trap, most probably with water leftover from the high humidity of the drift tube. The trapping time is $3 \mathrm{~ms}$, with a resonant excitation time of $15 \mathrm{~ms}$. Association reactions for trapped ions are not normally observed; this indicates that the $m / z 77$ ion reacts strongly and irreversibly with the low concentration of water in the trap. It can be suggested that the $m / z 77$ ion takes the form of a protonated benzyne ion after loss of the methyl group from the protonated toluene. The likelihood of this ion undergoing an irreversible association with water is easily imaginable in agreement with the likewise reaction between neutral benzyne and water to form phenol.

It is reasonable to assume the reaction in the ion funnel follows a similar route, whereby $m / z 77$ is first created by loss of the methyl group from the protonated toluene monomer and a later hydration reaction produces $m / z 95$ (see also Table 2 ). Although no ion at 
$m / z 77$ is detected from the ion funnel, it is conceivable that the abundance of water and the pace of the hydration reaction are such that the ion is so quickly converted to $m / z 95$, that the ion at $m / z 77$ is not long-lived enough for detection.

Comparing the level of fragmentation between the standard drift tube and the ion funnel for toluene and acetic acid it can be seen that for acetic acid a greater degree of total fragmentation is observed with the drift tube, but for toluene a greater degree of fragmentation is observed in the ion funnel. Gonzalez-Mendez et al. also find differences in fragmentation between the ion funnel and drift tube, differences which they go onto utilise for selectivity improvements [25]. The ion funnel generates many more collisions as compared to the drift tube, and the initial half of the reaction chamber is held at a very low reduced electric field in comparison to the standard drift tube. The low reduced electric field and high number of collisions in the RF section of the funnel may act to suppress proton transfer reaction induced dissociation and promote collision induced dissociation.

Acetic acid and alpha-pinene are both compounds which more readily undergo reaction with protonated water clusters to create protonated monomer product ions as compared to toluene or o-xylene [35]. In the case of alpha-pinene, a reaction with the protonated water cluster will probably proceed via direct proton transfer; alpha-pinene having a proton affinity of $878 \pm 8 \mathrm{~kJ} \mathrm{~mol}^{-1}$ [38] easily above either theoretical; $833 \mathrm{~kJ} \mathrm{~mol}^{-1}$ [39] or experimental; $808 \pm 6 \mathrm{~kJ} \mathrm{~mol}^{-1}$ [40] values for the water dimer. Acetic acid will react with the protonated water dimer via an intermediate cluster complex, often termed a ligand switch reaction in PTR-MS [41]. In the low reduced electric field region in the initial section of our ion funnel water clusters will dominate; in the funnel section itself clusters will be broken up by the RF electric field. Protonation reactions occurring in the low reduced electric field region will be of significantly lower kinetic energy than reactions in the ion funnel. Alpha-pinene and acetic acid will be more favorable to react in the low energy region of the reaction chamber and toluene and o-xylene in the high energy region. This could create more proton transfer induced dissociation in the o-xylene and toluene than in the acetic acid and alpha-pinene.

In the ion funnel an increase in product ion fragmentation is observed for increased RF voltages, although the increases are not as significant as the changes in branching ratios observed across operating voltages of the standard drift tube. Fragmentation in the ion funnel will occur at regions of high field, closest to the electric field plates. At higher RF fields the focusing is more pronounced, so the ions do not get as close to the plates. The increase in RF voltage affects the fragmentation, but perhaps not to the extent expected because the ion's trajectory is focused away from the high field region closest to the plates. This does not apply towards the exit of the ion funnel however, where ions are forced closer to the plates by the narrowing diameter of the plates. A reduction in the RF field is applied to the endmost plates to minimize this. Further to this, at increased dc voltages the ions trajectory can be altered to push it closer to the plates and into higher field regions, in this way the dc field controls the RF field experienced by an ion for a given RF voltage. The path taken by ions in the ion funnel shows a great deal of variation from the central axial path of the funnel, as shown in Fig. 2. At the extremity of the cone of ion traces, ions are at higher energy regions of the funnel than those ions taking a more central axial path. Given the high variation in the radial position of ions it is possible that product ions are subjected to various strengths of electric fields, for various durations of time. The large spread in spatial distribution of the ions and corresponding large spread in energy could be the cause of higher energy fragmentation pathways being accessible in toluene, while overall fragmentation is reduced for acetic acid.
The reagent ions will also be subject to this large spatial spread and corresponding energy spread. At higher energy the profile of the reagent ions will be directed more to favoring abundance of the monomer. In these higher energy regions closest to the plates the greatest chance of protonation will occur for molecules that have the highest dependence on the abundance of the reagent ion monomer (benzene, toluene).

Recent advances have been made in PTR-MS reaction chamber technology. Implementation of ions funnels have been shown by Barber et al. [22], Ferreira de Brito [23] and by commercial manufacturers of PTR-MS; Ionicon [24] and Kore Technology. Developments in reaction tube design also extend to non-funnel electrode arrangements with applied RF fields. Breitenlechner et al. [42] recently unveiled a novel reaction chamber design employing a three phase RF voltage to break up water clusters and a flow tube mechanism to convey ions to the end of the drift tube, thus effectively decoupling the ion's axial velocity from its kinetic energy and allowing for much longer interaction times at axially consistent reduced electric fields.

When using ion funnel technology as a reaction chamber, the change from a standard drift tube with reduced electric fields $(\mathrm{E} / \mathrm{N})$ to an ion funnel set-up gives a wealth of new information, interpretation and possibilities for manipulation of molecular reactions, as shown by the fragmentation data in Table 2 and in the work by Gonzalez-Mendez et al. [25]. The electric field of the ion is dependent on the ion's position and this moves the fragmentation away from a standard drift field approximation. The focusing effect of the ion funnel remains in evidence, and implementation of this device in a standard PTR-MS instrumentation is of valued importance. In the quest for improving sensitivity by increasing path length and interaction time the ion funnel provides obvious improvements as evidenced in the sensitivity results and SIMION calculations.

\section{Conclusion}

Using an ion funnel reaction chamber in PTR-MS has shown significant and compound specific improvements in sensitivity. The mechanism behind these improvements is investigated in this paper with a combination of SIMION simulations and experiments and is found to rely on an increase in reaction path length and thus reaction time, and additional focusing over the standard dc only drift tube. By clarifying the mechanism behind the improvements it is possible that future redesigns can work more efficiently to maximize product ion yield. Characterization of the funnel is shown for a range of compounds, illustrating peak sensitivity and product ion branching ratios, these are both important factors to consider when utilizing an ion funnel reaction chamber in a real world scenario.

\section{Acknowledgements}

This research received funding from the Netherlands Organization for Scientific Research (NWO) in the framework of the Technology Area COAST; project: Breath Volatiles; 053.21.103.

\section{Appendix A. Supplementary data}

Supplementary data associated with this article can be found, in the online version, at http://dx.doi.org/10.1016/j.ijms.2017.01.001.

\section{References}

[1] R.S. Blake, P.S. Monks, A.M. Ellis, Proton-transfer reaction mass spectrometry, Chem. Rev. 109 (2009) 861-896.

[2] W. Lindinger, A. Hansel, A. Jordan, On-line monitoring of volatile organic compounds at pptv levels by means of Proton-Transfer-Reaction Mass Spectrometry (PTR-MS) Medical applications, food control and environmental research, Int. J. Mass Spectom. Ion Process. (1998) 191-241. 
[3] M. Camredon, J.F. Hamilton, M.S. Alam, K.P. Wyche, T. Carr, I.R. White, P.S. Monks, A.R. Rickard, W.J. Bloss, Distribution of gaseous and particulate organic composition during dark alpha-pinene ozonolysis, Atmos. Chem. Phys. 10 (2010) 2893-2917.

[4] J. de Gouw, C. Warneke, Measurements of volatile organic compounds in the earths atmosphere using proton-transfer-reaction mass spectrometry, Mass Spectrom. Rev. 26 (2007) 223-257.

[5] J. de Gouw, C. Warneke, T. Karl, G. Eerdekens, C. van der Veen, R. Fall, Sensitivity and specificity of atmospheric trace gas detection by proton-transfer-reaction mass spectrometry, Int. J. Mass Spectrom. 223 (2003) 365-382.

[6] E. Szymańska, P.A. Brown, A. Ziere, S. Martins, M. Batenburg, F.J.M. Harren, L.M.C. Buydens, Comprehensive data scientific procedure for enhanced analysis and interpretation of real-time breath measurements in In vivo aroma-release studies, Anal. Chem. 87 (2015) 10338-10345.

[7] E.S. Holm, A.P.S. Adamsen, A. Feilberg, A. Schafer, M.M. Lokke, M.A. Petersen, Quality changes during storage of cooked and sliced meat products measured with PTR-MS and HS-GC-MS, Meat Sci. 95 (2013) 302-310.

[8] F. Biasioli, C. Yeretzian, F. Gasperi, T.D. Mark, PTR-MS monitoring of VOCs and BVOCs in food science and technology, Trac-Trends Anal. Chem. 30 (2011) 968-977.

[9] D. Samudrala, B. Geurts, P. Brown, E. Szymańska, J. Mandon, J. Jansen, L. Buydens, F.M. Harren, S. Cristescu, Changes in urine headspace composition as an effect of strenuous walking, Metabolomics (2015) 1-11.

[10] H. Danner, P. Brown, E.A. Cator, F.J.M. Harren, N.M. van Dam, S.M. Cristescu, Aboveground and belowground herbivores synergistically induce volatile organic sulfur compound emissions from shoots but not from roots, J. Chem. Ecol. 41 (2015) 631-640.

[11] P. Trefz, M. Schmidt, P. Oertel, J. Obermeier, B. Brock, S. Kamysek, J. Dunkl, R. Zimmermann, J.K. Schubert, W. Miekisch, Continuous real time breath gas monitoring in the clinical environment by

proton-transfer-reaction-time-of-flight-mass spectrometry, Anal. Chem. 85 (2013) 10321-10329.

[12] R. Gonzalez-Mendez, D.F. Reich, S.J. Mullock, C.A. Corlett, C.A. Mayhew Development and use of a thermal desorption unit and proton transfer reaction mass spectrometry for trace explosive detection: determination of the instrumental limits of detection and an investigation of memory effects, Int. J. Mass Spectrom. 385 (2015) 13-18.

[13] M. Lanza, W.J. Acton, P. Sulzer, K. Breiev, S. Jurschik, A. Jordan, E. Hartungen, G. Hanel, L. Mark, T.D. Mark, C.A. Mayhew, Selective reagent ionisation-time of flight-mass spectrometry: a rapid technology for the novel analysis of blends of new psychoactive substances, J. Mass Spectrom. 50 (2015) 427-431.

[14] A. Hansel, A. Jordan, R. Holzinger, P. Prazeller, W. Vogel, W. Lindinger, Proton-Transfer Reaction Mass Spectrometry: on-line trace gass analysis at the ppbv level, Int. J. Mass Spectrom. 149 (1995) 609-619.

[15] E. Hartungen, S. Jürschik, A. Jordan, A. Edtbauer, S. Feil, G. Hanel, H. Seehauser, S. Haidacher, R. Schottkowsky, L. Märk, S. Jaksch, B. Agarwal, K. Becker, C.A. Mayhew, P. Sulzer, T.D. Märk, Proton transfer reaction-mass spectrometry: fundamentals recent advances and applications, Eur. Phys. J. - Appl. Phys. 61 (2013), null-null.

[16] R.S. Blake, C. Whyte, C.O. Hughes, A.M. Ellis, P.S. Monks, Demonstration of proton-transfer reaction time-of-flight mass spectrometry for real-time analysis of trace volatile organic compounds, Anal. Chem. 76 (2004) 3841-3845.

[17] C.J. Ennis, J.C. Reynolds, B.J. Keely, L.J. Carpenter, A hollow cathode proton transfer reaction time of flight mass spectrometer, Int. J. Mass Spectrom. 247 (2005) 72-80.

[18] M.M.L. Steeghs, C. Sikkens, E. Crespo, S.M. Cristescu, F.J.M. Harren, Development of a proton-transfer reaction ion trap mass spectrometer: online detection and analysis of volatile organic compounds, Int. J. Mass Spectrom. 262 (2007) 16-24.

[19] S. Jürschik, A. Tani, P. Sulzer, S. Haidacher, A. Jordan, R. Schottkowsky, E. Hartungen, G. Hanel, H. Seehauser, L. Märk, T.D. Märk, Direct aqueous injection analysis of trace compounds in water with proton-transfer-reaction mass spectrometry (PTR-MS), Int. J. Mass Spectrom. 289 (2010) 173-176.

[20] S. Jurschik, P. Sulzer, F. Petersson, C.A. Mayhew, A. Jordan, B. Agarwal, S. Haidacher, H. Seehauser, K. Becker, T.D. Mark, Proton transfer reaction mass spectrometry for the sensitive and rapid real-time detection of solid high explosives in air and water, Anal. Bioanal. Chem. 398 (2010) 2813-2820.

[21] D. Materic, M. Lanza, P. Sulzer, J. Herbig, D. Bruhn, C. Turner, N. Mason, V. Gauci, Monoterpene separation by coupling proton transfer reaction time-of-flight mass spectrometry with fastGC, Anal. Bioanal. Chem. 407 (2015) 7757-7763.

[22] S. Barber, R.S. Blake, L.R. White, P.S. Monks, F. Reich, S. Mullock, A.M. Ellis, Increased sensitivity in proton transfer reaction mass spectrometry by incorporation of a radio frequency ion funnel, Anal. Chem. 84 (2012) 5387-5391.
[23] J. Ferreira de Brito, A Lightweight High-sensitivity Chemical Mass Spectrometer for Organic Compounds, Institut für Meteorologie und Klimaforschung (IMK), Karlsruhe Institut für Technologie (KIT), Karlsruhe, 2012.

[24] A. Jordan, S. Feil, P. Mutschlechner, G. Hanel, E. Hartungen, J. Herbig, L. Mark, P. Sulzer, S. Jurschik, S. Jaksch, Improving the sensitivity of proton transfer reaction - time-of-Flight - mass spectrometry (PTR-TOFMS), in: A. Hansel, J. Dunkl (Eds.), 7th International Conference on Proton Transfer Reaction Mass Spectrometry and Its Applications, Innsbruck University Press, Obergurgl, Austria, 2016, pp. 147-150.

[25] R. González-Méndez, P. Watts, D. Olivenza-León, D.F. Reich, S.J. Mullock, C.A. Corlett, S. Cairns, P. Hickey, M. Brookes, C.A. Mayhew, Enhancement of compound selectivity using a radio frequency ion-funnel proton transfer reaction mass spectrometer: improved specificity for explosive compounds, Anal. Chem. 88 (2016) 10624-10630.

[26] S.A. Shaffer, K.Q. Tang, G.A. Anderson, D.C. Prior, H.R. Udseth, R.D. Smith, A novel ion funnel for focusing ions at elevated pressure using electrospray ionization mass spectrometry, Rapid Commun. Mass Spectrom. 11 (1997) 1813-1817.

[27] S.A. Shaffer, D.C. Prior, G.A. Anderson, H.R. Udseth, R.D. Smith, An ion funnel interface for improved ion focusing and sensitivity using electrospray ionization mass spectrometry, Anal. Chem. 70 (1998) 4111-4119.

[28] S.A. Shaffer, A. Tolmachev, D.C. Prior, G.A. Anderson, H.R. Udseth, R.D. Smith, Characterization of an improved electrodynamic ion funnel interface for electrospray ionization mass spectrometry, Anal. Chem. 71 (1999) 2957-2964.

[29] D. Manura, SIMION HS1 Collision Model, Scientific Instrument Services Inc, 2007.

[30] E. Boamfa, M. Steeghs, S. Cristescu, F. Harren, Trace gas detection from fermentation processes in apples; an intercomparison study between proton-transfer-reaction mass spectrometry and laser photoacoustics, Int. J. Mass spectrom. 239 (2004) 193-201

[31] P. Sulzer, E. Hartungen, G. Hanel, S. Feil, K. Winkler, P. Mutschlechner, S. Haidacher, R. Schottkowsky, D. Gunsch, H. Seehauser, M. Striednig, S. Jürschik, K. Breiev, M. Lanza, J. Herbig, L. Märk, T.D. Märk, A. Jordan, A proton transfer reaction-quadrupole interface time-of-flight mass spectrometer (PTR-QiTOF): high speed due to extreme sensitivity, Int. J. Mass Spectrom. 368 (2014) 1-5.

[32] P. Brown, P. Watts, T.D. Mark, C.A. Mayhew, Proton transfer reaction mass spectrometry investigations on the effects of reduced electric field and reagent ion internal energy on product ion branching ratios for a series of saturated alcohols, Int. J. Mass Spectrom. 294 (2010) 103-111.

[33] D. Samudrala, P.A. Brown, J. Mandon, S.M. Cristescu, F.J.M. Harren, Optimization and sensitive detection of sulfur compounds emitted from plants using proton transfer reaction mass spectrometry, Int. J. Mass Spectrom. 386 (2015) 6-14.

[34] H.R. Shamlouei, M. Tabrizchi, Transmission of different ions through a drift tube, Int. J. Mass spectrom. 273 (2008) 78-83.

[35] C. Warneke, C. van der Veen, S. Luxembourg, J.A. de Gouw, A. Kok, Measurements of benzene and toluene in ambient air using proton-transfer-reaction mass spectrometry: calibration, humidity dependence, and field intercomparison, Int. J. Mass Spectrom. 207 (2001) $167-182$.

[36] T.J. Christian, B. Kleiss, R.J. Yokelson, R. Holzinger, P.J. Crutzen, W.M. Hao, T. Shirai, D.R. Blake, Comprehensive laboratory measurements of biomass-burning emissions: 2. First intercomparison of open-path FTIR, PTR-MS, and GC-MS/FID/ECD, J. Geophys. Res: Atmos. 109 (2004), n/a-n/a.

[37] S.D. Maleknia, T.L. Bell, M.A. Adams, PTR-MS analysis of reference and plant-emitted volatile organic compounds, Int. J. Mass Spectrom. 262 (2007) 203-210.

[38] T. Solouki, J.E. Szulejko, Bimolecular and unimolecular contributions to the disparate self-chemical ionizations of $\alpha$-Pinene and camphene isomers, J. Am. Soc. Mass Spectrom. 18 (2007) 2026-2039.

[39] Y. Kawai, O. Yamaguchi, K. Takeuchi, Y. Yamauchi, S. Ozawa, H. Nakai, Reactions of Protonated water clusters $\mathrm{H}+(\mathrm{H} 2 \mathrm{O}) \mathrm{n}$ (n=1-6) with dimethylsulfoxide in a guided ion beam apparatus, Chem. Phys. Lett. (2003) 69-73.

[40] D.J. Goebbert, P.G. Wentold, Water dimer proton affinity from the kinetic method: dissociation energy of the water dimer, Eur. J. Mass Spectrom. (Chichester, England) 10 (2004) 837-846.

[41] A. Pysanenko, P. Španěl, D. Smith, Analysis of the isobaric compounds propanol, acetic acid and methyl formate in humid air and breath by selected ion flow tube mass spectrometry SIFT-MS, Int. J. Mass Spectrom. 285 (2009) 42-48.

[42] M. Breitenlechner, L. Fischer, M. Hainer, A. Hansel, The PTR3: a novel unltra-high sensitivity PTR-ToF, in: A. Hansel, J. Dunkl (Eds.), 7th International Conference on Proton Transfer Reaction Mass Spectrometry and Its Applications, Innsbruck University Press, Obergurgl, Austria, 2016, pp. 138-139. 